\title{
Combinatorics of the asymmetric exclusion process on a semi-infinite lattice*
}

\author{
TOMOHIRO SASAmoto AND LAUREN Williams
}

\begin{abstract}
We study two versions of the asymmetric exclusion process (ASEP) - an ASEP on a semi-infinite lattice $\mathbb{Z}^{+}$with an open left boundary, and an ASEP on a finite lattice with open left and right boundaries - and we demonstrate a surprising relationship between their stationary measures. The semi-infinite ASEP was first studied by Liggett [7] and then Grosskinsky [6], while the finite ASEP had been introduced earlier by Spitzer [9] and Macdonald-GibbsPipkin [8]. We show that the finite correlation functions involving the first $L$ sites for the stationary measures on the semi-infinite ASEP can be obtained as a nonphysical specialization of the stationary distribution of an ASEP on a finite one-dimensional lattice with $L$ sites. Namely, if the output and input rates of particles at the right boundary of the finite ASEP are $\beta$ and $\delta$, respectively, and we set $\delta=-\beta$, then this specialization corresponds to sending the right boundary of the lattice to infinity. Combining this observation with work of the second author and Corteel $[2,3]$, we obtain a combinatorial formula for finite correlation functions of the ASEP on a semi-infinite lattice.
\end{abstract}

\section{Introduction}

The asymmetric exclusion process (ASEP) is a model in which particles hop on a lattice, subject to the condition that there is at most one particle per site. It was first introduced by Spitzer [9], and also by Macdonald-GibbsPipkin [8] in the context of protein synthesis, who studied this model on a finite lattice of $L$ sites. A version of the model where particles hop on the semi-infinite lattice $\mathbb{Z}^{+}$was studied by Liggett [7] and subsequently by Grosskinsky in his thesis [6]. In the semi-infinite ASEP, particles may enter and exit at the left boundary at rates $\alpha$ and $\gamma$, respectively, and in

arXiv: 1204.1114

*T.S. would like to acknowledge the financial support from KAKENHI (2274054, 25103004). L.W. is partially supported by an NSF CAREER award and an Alfred Sloan Fellowship. 

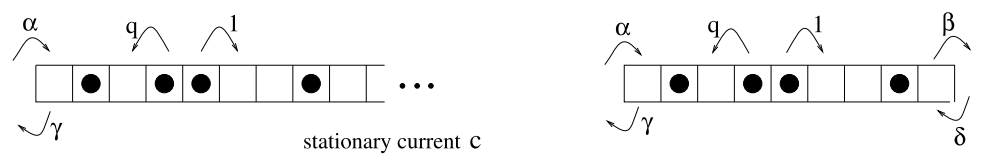

Figure 1.
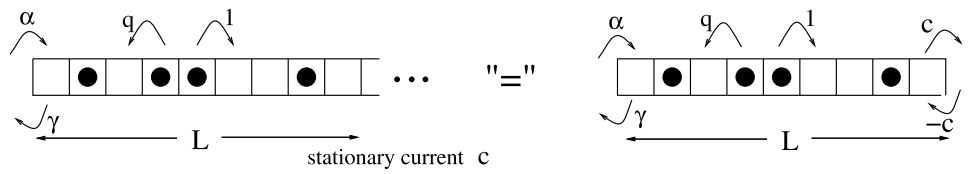

Figure 2.

the bulk, particles may hop right and left to neighboring sites of the lattice at rates 1 and $q$. Let $c$ be an additional parameter; it winds up determining the stationary current of particles. We denote states by vectors $\eta=\left(\eta_{1}, \eta_{2}, \ldots\right)$, where $\eta_{i} \in\{0,1\}$, and we denote the set of all states by $X$.

In the ASEP on a finite one-dimensional lattice of $L$ sites, particles may enter and exit at the left boundary at rates $\alpha$ and $\gamma$, and may exit and enter at the right boundary at rates $\beta$ and $\delta$. In the bulk, particles may hop right and left to neighboring sites of the lattice at rates 1 and $q$.

We refer to these two flavors of the ASEP as the semi-infinite ASEP and the finite ASEP. The two models are illustrated in Figure 1. In both models we assume that all parameters are non-negative.

Given a measure $\mu$ on $X$, and a word $\left(\eta_{1}, \ldots, \eta_{L}\right) \in\{0,1\}^{L}$, the correlation function $\left\langle\eta_{1} \ldots \eta_{L}\right\rangle$ is the expected value with respect to $\mu$ that the leftmost $L$ sites of a state in the semi-infinite ASEP will be $\eta_{1}, \ldots, \eta_{L}$.

Our first result is the following.

Theorem 1.1. The finite correlation functions involving the leftmost $L$ sites of the stationary measures of the semi-infinite ASEP can be obtained from the stationary distribution for the finite ASEP on a lattice of $L$ sites, after setting $\beta=c$ and $\delta=-c$. More specifically, the correlation function $\left\langle\eta_{1} \ldots \eta_{L}\right\rangle$ of the stationary measure for the semi-infinite ASEP corresponding to the stationary current $c$ is equal to $\mu^{\text {fin }}(\alpha, c, \gamma,-c ; q)\left(\eta_{1}, \ldots, \eta_{L}\right)$, the quantity one obtains by setting $\beta=c$ and $\delta=-c$ in the steady state probability of state $\left(\eta_{1}, \ldots, \eta_{L}\right)$ in the finite ASEP.

This theorem is illustrated in Figure 2. 

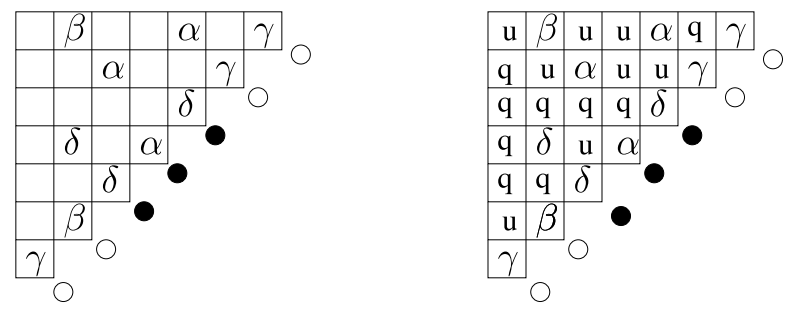

Figure 3: A staircase tableau of size 7 and type $\circ \circ$

By combining Theorem 1.1 with work of the second author and Corteel $[2,3]$ we can give a combinatorial formula for the finite correlation functions for the stationary measures for the semi-infinite ASEP. Before stating the result, we first introduce the staircase tableaux from $[2,3]$.

Definition 1.2. A staircase tableau of size $L$ is a Young diagram of "staircase" shape $(L, L-1, \ldots, 2,1)$ such that boxes are either empty or labeled with $\alpha, \beta, \gamma$, or $\delta$, subject to the following conditions:

- no box along the diagonal is empty;

- all boxes in the same row and to the left of a $\beta$ or a $\delta$ are empty;

- all boxes in the same column and above an $\alpha$ or a $\gamma$ are empty.

The type of a staircase tableau is a word in $\{o, \bullet\}^{L}$ obtained by reading the diagonal boxes from northeast to southwest and writing a $\bullet$ for each $\alpha$ or $\delta$, and $\mathrm{a} \circ$ for each $\beta$ or $\gamma$.

See the left of Figure 3 for an example of a staircase tableau.

Definition 1.3. The weight $\operatorname{wt}(\mathcal{T})$ of a staircase tableau $\mathcal{T}$ is a monomial in $\alpha, \beta, \gamma, \delta, q$, and $u$, which we obtain as follows. Every blank box of $\mathcal{T}$ is assigned a $q$ or $u$, based on the label of the closest labeled box to its right in the same row and the label of the closest labeled box below it in the same column, such that:

- every blank box which sees a $\beta$ to its right gets assigned a $u$;

- every blank box which sees a $\delta$ to its right gets assigned a $q$;

- every blank box which sees an $\alpha$ or $\gamma$ to its right, and an $\alpha$ or $\delta$ below it, gets assigned a $u$;

- every blank box which sees an $\alpha$ or $\gamma$ to its right, and a $\beta$ or $\gamma$ below it, gets assigned a $q$.

After assigning a $q$ or $u$ to each blank box in this way, the weight of $\mathcal{T}$ is then defined as the product of all labels in all boxes. 


\begin{tabular}{|c|c|c|c|c|c|c|c|c|c|c|c|c|c|c|}
\hline $\begin{array}{lll}u & a\end{array}$ & $\chi$ & $\delta$ & $q$ & $\delta$ & $u$ & $\alpha$ & $\alpha$ & $\alpha$ & & $\alpha$ & & & & \\
\hline$\alpha$ & $\delta$ & & $\alpha$ & & $\delta$ & & $\delta$ & & O & & & & & $\delta$ \\
\hline
\end{tabular}

Figure 4: The tableaux of type

The right of Figure 3 shows that this staircase tableau has weight $\alpha^{3} \beta^{2} \gamma^{3} \delta^{3} q^{9} u^{8}$.

Remark 1.4. The weight of a staircase tableau of size $L$ has degree $L(L+$ $1) / 2$. We will typically set $u=1$. Keeping $u$ general corresponds to particles in the bulk hopping right at rate $u$ instead of 1 .

The following result, concerning the stationary distribution of the finite ASEP, was announced in [2] and proved in [3].

Theorem $1.5([2,3])$. Consider any state $\tau$ of the finite ASEP with L sites, where the parameters $\alpha, \beta, \gamma, \delta, q$, u are general. Set $Z_{L}^{\text {fin }}=\sum_{\mathcal{T}} \mathrm{wt}(\mathcal{T})$, where the sum is over all staircase tableaux of size $L$; we call $Z_{L}^{\text {fin }}$ the partition function of the finite ASEP. Then the steady state probability that the ASEP is at state $\tau$ is precisely

$$
\frac{\sum_{\mathcal{T}} \mathrm{wt}(\mathcal{T})}{Z_{L}^{\text {fin }}}
$$

where the sum is over all staircase tableaux $\mathcal{T}$ of type $\tau$.

Example 1.6. Figure 4 illustrates Theorem 1.5 for the state $\bullet \bullet$ of the ASEP. All staircase tableaux $\mathcal{T}$ of type $\bullet \bullet$ are shown. It follows that the steady state probability of $\bullet \bullet$ is

$$
\frac{\alpha^{2} u+\delta^{2} q+\alpha \delta q+\alpha \delta u+\alpha^{2} \delta+\alpha \beta \delta+\alpha \gamma \delta+\alpha \delta^{2}}{Z_{2}^{f i n}} .
$$

Remark 1.7. In [1], the second author, together with Corteel, Stanley, and Stanton, studied staircase tableaux and their generating function $Z_{L}^{\text {fin }}(\alpha, \beta$, $\gamma, \delta ; q)$ from a combinatorial point of view. In particular, Table 1 of [1] lists various specializations of $Z_{L}^{\text {fin }}(\alpha, \beta, \gamma, \delta ; q)$. The third row of the table shows that

$$
Z_{L}^{f i n}(\alpha, \beta, \gamma,-\beta ; q)=\prod_{j=0}^{L-1}\left(\alpha+q^{j} \gamma\right)
$$


Note that despite the fact that the specialization is nonphysical (we have made the hopping rate $\delta$ a negative real number), the resulting quantity is positive. Also, the resulting quantity has no dependence on $\beta$ and $\delta$.

Corollary 1.8. Consider the semi-infinite ASEP, with parameters $\alpha, \gamma, q, c$. Set $Z_{L}^{\text {semi }}=\prod_{j=0}^{L-1}\left(\alpha+q^{j} \gamma\right)$. Then the correlation function $\left\langle\eta_{1} \ldots \eta_{L}\right\rangle$ for the stationary measure is precisely

$$
\frac{\left.\sum_{\mathcal{T}} \operatorname{wt}(\mathcal{T})\right|_{u=1, \beta=c, \delta=-c}}{Z_{L}^{\text {semi }}},
$$

where the sum is over all staircase tableaux $\mathcal{T}$ of type $\left(\eta_{1}, \ldots, \eta_{L}\right)$.

Proof. Corollary 1.8 follows from Theorem 1.1, Theorem 1.5, and Remark 1.7.

Remark 1.9. For the finite ASEP these correlation functions could be written as polynomials in the parameters with all coefficients being positive (divided by a normalization factor). However, for the semi-infinite ASEP, since we use the substition $\delta=-c$ in Corollary 1.8, this positivity property of the coefficients no longer holds.

Nevertheless, in Theorem 5.1 we will provide a sufficient condition for the quantities in (1) to be positive real numbers.

Example 1.10. We can use Corollary 1.8 and the tableaux of Figure 4 to compute the correlation function $\left\langle\eta_{1} \eta_{2}\right\rangle=\langle 11\rangle$. Setting $u=1, \beta=c, \delta=-c$ gives

$$
\begin{gathered}
\frac{\alpha^{2}+c^{2} q-\alpha c q-\alpha c-\alpha^{2} c-\alpha c^{2}-\alpha \gamma c+\alpha c^{2}}{(\alpha+\gamma)(\alpha+q \gamma)} \\
=\frac{\alpha^{2}+c^{2} q-\alpha c q-\alpha c-\alpha^{2} c-\alpha \gamma c}{(\alpha+\gamma)(\alpha+q \gamma)}
\end{gathered}
$$

The structure of this paper is as follows. In Section 2 we review some results on the finite ASEP, then in Section 3 we define the ASEP on a semiinfinite lattice. In Section 4 we state and prove a matrix ansatz describing the finite correlation functions of its (signed) stationary measures, and in Section 5 we provide a sufficient condition for these signed stationary measures to be positive. In Section 6 we prove Theorem 1.1. And in Section 7 we summarize our results and end with some questions about "nonsensical" specializations of Markov chains.

Acknowledgments: The authors are grateful to A. Borodin, P. Deift, P.L. Ferrari, S. Grosskinsky, and H. Spohn for useful discussions. 


\section{Background on the finite ASEP and its matrix ansatz}

We start by recalling the Matrix Ansatz of Derrida, Evans, Hakim, and Pasquier [4] for the finite ASEP, as well as results of the first author together with Uchiyama and Wadati [10] on the current.

Theorem $2.1([4])$. Suppose that there are matrices $D, E$ and vectors $\langle W|$, $|V\rangle$, which satisfy

(A.) $D E-q E D=D+E$

(B.) $\alpha\langle W| E-\gamma\langle W| D=\langle W|$

(C.) $\beta D|V\rangle-\delta E|V\rangle=|V\rangle$.

Let $\eta=\left(\eta_{1}, \ldots, \eta_{L}\right)$ be a state of the finite ASEP. Then the measure $\mu$ defined by

$$
\mu(\eta)=\frac{\left\langle W\left|\prod_{x=1}^{L} \eta_{x} D+\left(1-\eta_{x}\right) E\right| V\right\rangle}{\left\langle W\left|(D+E)^{L}\right| V\right\rangle}
$$

is the unique stationary measure for the ASEP on a finite lattice of $L$ sites, where the rates of particles entering and exiting at the left are $\alpha$ and $\gamma$, and the rates of particles exiting and entering at the right are $\beta$ and $\delta$.

Although Theorem 2.1 was published in 1993, it was not until ten years later that a general solution to the ansatz was obtained.

Theorem 2.2 ([10, Section 4.2]). There is a solution $D, E,\langle W|| V$,$\rangle which$ satisfies the relations of Theorem 2.1.

The above solution was related to Askey-Wilson polynomials. Using properties of the Askey-Wilson integral, the authors calculated the current $J_{L}$ of the finite ASEP. Recall that $J_{L}=\frac{Z_{L-1}}{Z_{L}}$, where $Z_{L}=\left\langle W\left|(D+E)^{L}\right| V\right\rangle$. Let $J=\lim _{L \rightarrow \infty} J_{L}$.

Proposition 2.3 ([10, (6.5), (6.8), (6.11)]). Suppose that $q \neq 1$. Let

$$
\begin{aligned}
& a=\frac{1-q-\alpha+\gamma+\sqrt{(1-q-\alpha+\gamma)^{2}+4 \alpha \gamma}}{2 \alpha} \text { and } \\
& b=\frac{1-q-\beta+\delta+\sqrt{(1-q-\beta+\delta)^{2}+4 \beta \delta}}{2 \beta} .
\end{aligned}
$$

1. If $a>1$ and $a>b$ then $J=(1-q) \frac{a}{(1+a)^{2}}$.

2. If $a<1$ and $b<1$ then $J=\frac{1-q}{4}$.

3. If $b>1$ and $b>a$ then $J=(1-q) \frac{b}{(1+b)^{2}}$. 


\section{Formal definition of the semi-infinite ASEP}

We now define the semi-infinite ASEP. Since this is a Markov process with infinitely many states, one must define it carefully; we give its Markov generator below. This Markov generator then determines a Markov semigroup and hence a Markov process, see [7, Chapter 1] or [6, Section A.1] for details.

Let $\eta=\left(\eta_{1}, \eta_{2}, \ldots\right)$ be a state in $X$. If $i$ is a positive integer, we define from $\eta$ two new states $\eta^{i}$ and $\eta^{i, i+1}$ by

$$
\begin{aligned}
\left(\eta^{i}\right)_{j}= & \begin{cases}1-\eta_{i} & \text { if } j=i \\
\eta_{j} & \text { if } j \neq i\end{cases} \\
\text { and }\left(\eta^{i, i+1}\right)_{j} & = \begin{cases}\eta_{i+1} & \text { if } j=i \\
\eta_{i} & \text { if } j=i+1 \\
\eta_{j} & \text { if } j \neq i, i+1\end{cases}
\end{aligned}
$$

Let $C_{0}(X)$ be the set of cylinder functions on $X$, i.e. functions from $X$ to $\mathbb{R}$ which depend on only finitely many sites.

Definition 3.1. The Markov generator $\mathcal{L}$ of the semi-infinite ASEP is defined as follows. Given any function $f \in C_{0}(X)$,

$$
\begin{aligned}
\mathcal{L} f(\eta)= & \alpha\left(1-\eta_{1}\right)\left(f\left(\eta^{1}\right)-f(\eta)\right)+\gamma \eta_{1}\left(f\left(\eta^{1}\right)-f(\eta)\right) \\
& +\sum_{x=1}^{\infty}\left(\eta_{x}\left(1-\eta_{x+1}\right)\left(f\left(\eta^{x, x+1}\right)-f(\eta)\right)\right. \\
& \left.+q\left(1-\eta_{x}\right) \eta_{x+1}\left(f\left(\eta^{x, x+1}\right)-f(\eta)\right)\right) .
\end{aligned}
$$

We are interested in stationary measures of the corresponding Markov process. A measure $\mu$ is stationary if $\mathbb{E}^{\mu}(\mathcal{L} f)=0$ for all $f \in C_{0}(X)$. Here $\mathbb{E}^{\mu}$ is the expected value with respect to a measure $\mu$. Note that since the state space $X$ is infinite, the uniqueness of the stationary measure is no longer assured.

\section{The matrix ansatz for the semi-infinite ASEP}

We first prove a matrix ansatz in the spirit of [4]. The version which we shall state and prove for the semi-infinite ASEP is a generalization of a theorem of Grosskinsky [6, Theorem 3.2]; we recover his ansatz if we set $\gamma=0$ and $q=0$. Note that although our proof is quite analogous to Grosskinsky's proof, he omits the details of the calculations, so we give a full proof below. 
Prior to Grosskinsky's work, the paper [5] observed that one can adapt the matrix ansatz to compute cylindrical functions on semi-infinite and infinite lines.

In what follows, we use the terminology signed measure for a measure which is not necessarily positive. We will first give a matrix ansatz which describes stationary signed measures (Theorem 4.1), and then in the following section, we'll give a theorem (Theorem 5.1) which provides conditions guaranteeing that the measures are positive.

Theorem 4.1. Suppose there are matrices $D, E$ and vectors $\langle W|| V$,$\rangle , which$ satisfy

(a.) $D E-q E D=c(D+E)$

(b.) $\alpha\langle W| E-\gamma\langle W| D=c\langle W|$

(c.) $(D+E)|V\rangle=|V\rangle$.

Let $\eta=\left(\eta_{1}, \eta_{2}, \ldots, \eta_{L}\right) \in\{0,1\}^{L}$. Then the signed measure $\mu^{L}$ defined by

$$
\mu^{L}\left(\eta_{1}, \ldots, \eta_{L}\right)=\frac{\left\langle W\left|\prod_{x=1}^{L} \eta_{x} D+\left(1-\eta_{x}\right) E\right| V\right\rangle}{\left\langle W\left|(D+E)^{L}\right| V\right\rangle}
$$

is stationary for the process defined by $\mathcal{L}$. Here the parameter $c$ determines the stationary current, i.e. $\mathbb{E}^{\mu}\left(\eta_{x}\left(1-\eta_{x+1}\right)-q\left(1-\eta_{x}\right) \eta_{x+1}\right)=c$ for all $x \in \mathbb{Z}^{+}$.

Remark 4.2. Note that the relation (c.) above is essentially a special case of the relation (C.) from Theorem 2.1, after setting $\delta=-\beta$. In Proposition 6.1, we will exploit this coincidence.

Remark 4.3. The measure $\mu^{L}$ defined above does not depend on the choice of solution $D, E,\langle W|| V$,$\rangle . Indeed, for any word Y$ in $D$ and $E$, by repeatedly applying relations (a.), (b.) and (c.), one can express $\langle W|Y| V\rangle$ in terms of $\alpha, \gamma, q, c$, and $\langle W \mid V\rangle$.

Proof. Suppose that $f \in C_{0}(X)$ concentrates on sites $\{1,2, \ldots, L\}$. Using Definition 3.1, the stationary condition which we must check becomes:

$$
\begin{aligned}
0= & \sum_{\eta} \alpha \mu^{L}(\eta)\left(1-\eta_{1}\right)\left(f\left(\eta^{1}\right)-f(\eta)\right)+\sum_{\eta} \gamma \mu^{L}(\eta) \eta_{1}\left(f\left(\eta^{1}\right)-f(\eta)\right) \\
& +\sum_{\eta} \sum_{x=1}^{L-1}\left[\mu^{L}(\eta) \eta_{x}\left(1-\eta_{x+1}\right)\left(f\left(\eta^{x, x+1}\right)-f(\eta)\right)\right. \\
& \left.+q \mu^{L}(\eta)\left(1-\eta_{x}\right) \eta_{x+1}\left(f\left(\eta^{x, x+1}\right)-f(\eta)\right)\right]
\end{aligned}
$$




$$
\begin{aligned}
& +\sum_{\eta}\left[\mu^{L+1}(\eta) \eta_{L}\left(1-\eta_{L+1}\right)\left(f\left(\eta^{L, L+1}\right)-f(\eta)\right)\right. \\
& \left.+q \mu^{L+1}(\eta)\left(1-\eta_{L}\right) \eta_{L+1}\left(f\left(\eta^{L, L+1}\right)-f(\eta)\right)\right] .
\end{aligned}
$$

Here the sum is over all $\eta \in\{0,1\}^{L}$. Rewriting this equation gives

$$
\begin{aligned}
0= & \sum_{\eta} f(\eta)\left(\alpha \mu^{L}\left(\eta^{1}\right)\left(1-\eta_{1}^{1}\right)-\alpha \mu^{L}(\eta)\left(1-\eta_{1}\right)+\gamma \mu^{L}\left(\eta^{1}\right) \eta_{1}^{1}-\gamma \mu^{L}(\eta) \eta_{1}\right) \\
& +\sum_{x=1}^{L-1}\left[\mu^{L}\left(\eta^{x, x+1}\right) \eta_{x}^{x, x+1}\left(1-\eta_{x+1}^{x, x+1}\right)-\mu^{L}(\eta) \eta_{x}\left(1-\eta_{x+1}\right)\right. \\
& \left.+q \mu^{L}\left(\eta^{x, x+1}\right)\left(1-\eta_{x}^{x, x+1}\right) \eta_{x+1}^{x, x+1}-q \mu^{L}(\eta)\left(1-\eta_{x}\right) \eta_{x+1}\right] \\
& +\mu^{L+1}\left(\eta^{L, L+1}\right) \eta_{L}^{L, L+1}\left(1-\eta_{L+1}^{L, L+1}-\mu^{L+1}(\eta) \eta_{L}\left(1-\eta_{L+1}\right)\right. \\
& \left.+q \mu^{L+1}\left(\eta^{L, L+1}\right)\left(1-\eta_{L}^{L, L+1}\right) \eta_{L+1}^{L, L+1}-q \mu^{L+1}(\eta)\left(1-\eta_{L}\right) \eta_{L+1}\right)
\end{aligned}
$$

Note that $\eta_{1}^{1}=1-\eta_{1}$. The coefficient of $f(\eta)$ in the above equation is

$$
\begin{aligned}
& \sum_{x=1}^{L-1}\left[\mu^{L}\left(\eta^{x, x+1}\right) \eta_{x+1}\left(1-\eta_{x}\right)-\mu^{L}(\eta) \eta_{x}\left(1-\eta_{x+1}\right)\right. \\
& \left.\quad+q \mu^{L}\left(\eta^{x, x+1}\right)\left(1-\eta_{x+1}\right) \eta_{x}-q \mu^{L}(\eta)\left(1-\eta_{x}\right) \eta_{x+1}\right] \\
& \quad+\mu^{L+1}\left(\eta^{L, L+1}\right) \eta_{L+1}\left(1-\eta_{L}\right)-\mu^{L+1}(\eta) \eta_{L}\left(1-\eta_{L+1}\right) \\
& \quad+q \mu^{L+1}\left(\eta^{L, L+1}\right)\left(1-\eta_{L+1}\right) \eta_{L}-q \mu^{L+1}(\eta)\left(1-\eta_{L}\right) \eta_{L+1} \\
& \quad+\alpha \mu^{L}\left(\eta^{1}\right) \eta_{1}-\alpha \mu^{L}(\eta)\left(1-\eta_{1}\right)+\gamma \mu^{L}\left(\eta^{1}\right)\left(1-\eta_{1}\right)-\gamma \mu^{L}(\eta) \eta_{1}
\end{aligned}
$$

We aim to show that each coefficient is equal to 0 . Rearranging terms gives

$$
\begin{aligned}
& \sum_{x=1}^{L-1}\left[\mu^{L}\left(\eta^{x, x+1}\right) \eta_{x+1}\left(1-\eta_{x}\right)-q \mu^{L}(\eta)\left(1-\eta_{x}\right) \eta_{x+1}\right. \\
& \left.\quad-\left(\mu^{L}(\eta) \eta_{x}\left(1-\eta_{x+1}\right)-q \mu^{L}\left(\eta^{x, x+1}\right)\left(1-\eta_{x+1}\right) \eta_{x}\right)\right] \\
& \quad+\mu^{L+1}\left(\eta^{L, L+1}\right) \eta_{L+1}\left(1-\eta_{L}\right)-q \mu^{L+1}(\eta)\left(1-\eta_{L}\right) \eta_{L+1} \\
& \quad-\left[\mu^{L+1}(\eta) \eta_{L}\left(1-\eta_{L+1}\right)-q \mu^{L+1}\left(\eta^{L, L+1}\right)\left(1-\eta_{L+1}\right) \eta_{L}\right] \\
& \quad+\left[\alpha \mu^{L}\left(\eta^{1}\right) \eta_{1}-\gamma \mu^{L}(\eta) \eta_{1}\right]-\left[\alpha \mu^{L}(\eta)\left(1-\eta_{1}\right)-\gamma \mu^{L}\left(\eta^{1}\right)\left(1-\eta_{1}\right)\right]
\end{aligned}
$$

Note that each configuration of particles can be seen as a sequence of empty and occupied blocks. Suppose that the first $L$ sites of $\eta$ consists of 
$n$ such blocks $(\circ \cdots \circ)(\bullet \cdots \bullet)(\circ \cdots \circ) \ldots(\bullet \cdots \bullet)(\circ \cdots \circ)$ where there are $\tau_{1}$ o's in the first block, $\tau_{2} \bullet$ 's in the second block, ..., and $\tau_{n}$ o's in the last block. Here we assume that all $\tau_{i}$ 's are nonzero, so in particular, the first $L$ sites of $\eta$ begin and end with $\circ$. Thinking of the configuration of particles as a sequence of empty and occupied blocks, we also use $\tau$ to denote $\eta$.

At a boundary between a full and empty block $\left(\tau_{i}\right.$ and $\left.\tau_{i+1}\right)$ we can apply the bulk rule of the ansatz to get $\tau-q \tau^{\prime}=c\left(\tau^{i}+\tau^{i+1}\right)$. Here, $\tau^{\prime}$ is the configuration obtained from $\tau$ by swapping the adjacent $\bullet$ and $\circ$ in the $i$ th and $i+1$ st block, and $\tau^{i}$ is obtained from $\tau$ by deleting one site in block $i$.

Noting that it has nonzero values only at the block boundaries, the sum over $x$ in (3) and (4) telescopes:

$$
\begin{aligned}
& \sum_{i=1, i \text { odd }}^{n-2} c\left[\mu^{L-1}\left(\tau^{i}\right)+\mu^{L-1}\left(\tau^{i+1}\right)-\left(\mu^{L-1}\left(\tau^{i+1}\right)+\mu^{L-1}\left(\tau^{i+2}\right)\right)\right] \\
& \quad=c \mu^{L-1}\left(\tau^{1}\right)-c \mu^{L-1}\left(\tau^{n}\right)
\end{aligned}
$$

Since we have assumed that the first $L$ sites of $\eta$ begin and end with a $\circ$, we have that $\eta_{1}=\eta_{L}=0$. Applying this and the relations of the ansatz allows us to simplify the quantities (5), (6) and (7):

$$
\begin{aligned}
+\mu^{L+1}\left(\eta^{L, L+1}\right) \eta_{L+1}\left(1-\eta_{L}\right)-q \mu^{L+1}(\eta)\left(1-\eta_{L}\right) \eta_{L+1} & =c\left(\mu^{L}(\eta)+\mu^{L}\left(\eta^{L}\right)\right) \\
-\left[\mu^{L+1}(\eta) \eta_{L}\left(1-\eta_{L+1}\right)-q \mu^{L+1}\left(\eta^{L, L+1}\right)\left(1-\eta_{L+1}\right) \eta_{L}\right] & =0 \\
+\alpha \mu^{L}\left(\eta^{1}\right) \eta_{1}-\gamma \mu^{L}(\eta) \eta_{1} & =0 \\
-\left[\alpha \mu^{L}(\eta)\left(1-\eta_{1}\right)-\gamma \mu^{L}\left(\eta^{1}\right)\left(1-\eta_{1}\right)\right] & =-\alpha \mu^{L}(\eta) \\
& +\gamma \mu^{L}\left(\eta^{1}\right) .
\end{aligned}
$$

Therefore the coefficient of $f(\eta)$, which is given by (3) through (7), simplifies to

$$
c \mu^{L-1}\left(\tau^{1}\right)-c \mu^{L-1}\left(\tau^{n}\right)+c \mu^{L}(\eta)+c \mu^{L}\left(\eta^{L}\right)-\alpha \mu^{L}(\eta)+\gamma \mu^{L}\left(\eta^{1}\right) .
$$

But now note that by relation (c.) of the ansatz, $c \mu^{L}(\eta)+c \mu^{L}\left(\eta^{L}\right)=$ $c \mu^{L-1}\left(\tau^{n}\right)$, and by relation (b.) of the ansatz, $-\alpha \mu^{L}(\eta)+\gamma \mu^{L}\left(\eta^{1}\right)=$ $-c \mu^{L-1}\left(\tau^{1}\right)$. It follows that the coefficient of $f(\eta)$ is 0 .

This completes the proof, when the first $L$ sites of $\eta$ begin and end with $\circ$. The proof is analogous if the first $L$ sites begin or end with

Remark 4.4. In fact the above argument proves the following statement. Suppose that $g:\{D, E\}^{*} \rightarrow \mathbb{R}$ is a function on words in $D$ and $E$ (extended 
linearly to linear combinations of such words) such that for any words $Y$ and $Y^{\prime}$ in $D$ and $E$, we have the following:
(a.) $g\left(Y(D E-q E D) Y^{\prime}\right)=c g\left(Y(D+E) Y^{\prime}\right)$
(b.) $g(\alpha E Y-\gamma D Y)=c g(Y)$
(c.) $g(Y(D+E))=g(Y)$.

Let $\eta=\left(\eta_{1}, \eta_{2}, \ldots, \eta_{L}\right) \in\{0,1\}^{L}$. Then the signed measure $\mu^{L}$ defined by

$$
\mu^{L}\left(\eta_{1}, \ldots, \eta_{L}\right)=\frac{g\left(\prod_{x=1}^{L} \eta_{x} D+\left(1-\eta_{x}\right) E\right)}{g\left((D+E)^{L}\right)}
$$

is stationary for the process defined by $\mathcal{L}$. Here the parameter $c$ determines the stationary current, i.e. $\mathbb{E}^{\mu}\left(\eta_{x}\left(1-\eta_{x+1}\right)-q\left(1-\eta_{x}\right) \eta_{x+1}\right)=c$ for all $x \in \mathbb{Z}^{+}$.

\section{Positivity of the measures}

One would like to know when the signed measures defined in (2) or (8) is positive.

Theorem 5.1. The signed measure defined in (2) (equivalently, (8)) is positive provided that $q \leq 1$ and one of the inequalities below is satisfied:

1. $a \geq 1$ and $c \leq(1-q) a /(1+a)^{2}$, or

2. $a \leq 1$ and $c \leq(1-q) / 4$.

Here a is defined as in Proposition 2.3.

We will prove Theorem 5.1 by finding a solution to the semi-infinite matrix ansatz (the version in Remark 4.4) which is obtained as a limit of a solution to the finite matrix ansatz.

Proposition 5.2. Let $D, E,\langle W|| V$,$\rangle denote the solution to the finite$ matrix ansatz from Theorem 2.2. Let $C=D+E$. Then for any word $Y$ in $D$ and $E$, the following limit exists:

$$
\lim _{m \rightarrow \infty} \frac{\left\langle W\left|Y C^{m}\right| V\right\rangle}{\left\langle W\left|C^{m}\right| V\right\rangle} .
$$

Proof. We will use relations (A.) and (B.) of the finite matrix ansatz together with the fact (Proposition 2.3) that $\lim _{m \rightarrow \infty} \frac{\left\langle W\left|C^{m-1}\right| V\right\rangle}{\left\langle W\left|C^{m}\right| V\right\rangle}$ exists. Note that the latter fact implies that for any finite $\ell$, both $\lim _{m \rightarrow \infty} \frac{\left\langle W\left|C^{\ell} C^{m-1}\right| V\right\rangle}{\left\langle W\left|C^{m}\right| V\right\rangle}$ and $\lim _{m \rightarrow \infty} \frac{\left\langle W\left|C^{m-1}\right| V\right\rangle}{\left\langle W\left|C^{\ell} C^{m}\right| V\right\rangle}$ exist. 
We will prove the result by induction on the length of $Y$. We start by considering the length 1 case, i.e. $Y=D$ or $Y=E$. Let $x_{m}=\frac{\left\langle W\left|E C^{m}\right| V\right\rangle}{\left\langle W\left|C^{m+1}\right| V\right\rangle}$ and $y_{m}=\frac{\left\langle W\left|D C^{m}\right| V\right\rangle}{\left\langle W\left|C^{m+1}\right| V\right\rangle}$. Then we have $x_{m}+y_{m}=1$. But also, by relation (B.) of the ansatz, we have $\alpha x_{m}-\gamma y_{m}=\frac{Z_{m}}{Z_{m+1}}=J_{m+1}$. We can therefore solve for $x_{m}$ and $y_{m}$ in terms of $J_{m+1}$; since the limit of $J_{m+1}$ exists as $m \rightarrow \infty$, so does the limit of $x_{m}$ and $y_{m}$. It follows that for $Y=D$ or $Y=E$, the limit $\lim _{m \rightarrow \infty} \frac{\left\langle W\left|Y C^{m}\right| V\right\rangle}{\left\langle W\left|C^{m}\right| V\right\rangle}$ exists.

More generally, for any word $Y^{\prime}$ of length $\ell\left(Y^{\prime}\right)>1$, we will show that we can solve for $\frac{\left\langle W\left|Y^{\prime} C^{m}\right| V\right\rangle}{\left\langle W\left|C^{m}\right| V\right\rangle}$ in terms of quantities of the form $\frac{\left\langle W\left|Y C^{m}\right| V\right\rangle}{\left\langle W\left|C^{m}\right| V\right\rangle}$ where the length $\ell(Y)$ of $Y$ is at most $\ell\left(Y^{\prime}\right)-1$. This will complete the proof, since by the inductive hypothesis, we can write the latter quantities in terms of the parameters $\alpha, \beta, \gamma, \delta, q$ and $J_{m}$ 's, and hence can take the limit as $m$ goes to infinity.

Note that any word $Y^{\prime}$ of length greater than 1 can be written in the form $D Y$ or $E Y$ where the length of $Y$ is non-negative. Using relation (B.) of the finite matrix ansatz, for any word $Y$ in $D$ and $E$, we have that

$$
\alpha\left\langle W\left|E Y C^{m}\right| V\right\rangle-\gamma\left\langle W\left|D Y C^{m}\right| V\right\rangle=\left\langle W\left|Y C^{m}\right| V\right\rangle
$$

And by repeatedly using relation (A.) of the ansatz, we can write

$$
\begin{aligned}
q^{\ell(Y)}\left\langle W\left|E Y C^{m}\right| V\right\rangle & =q^{\# E^{\prime} s \text { in } Y}\left\langle W\left|Y E C^{m}\right| V\right\rangle+\text { terms of shorter length. } \\
\left\langle W\left|D Y C^{m}\right| V\right\rangle & =q^{\# E^{\prime} s \text { in } Y}\left\langle W\left|Y D C^{m}\right| V\right\rangle+\text { terms of shorter length. }
\end{aligned}
$$

Here a term of shorter length means a monomial in the parameters times a term of the form $\left\langle W\left|Y^{\prime \prime} C^{m}\right| V\right\rangle$ where $\ell\left(Y^{\prime \prime}\right)<\ell(Y E)=\ell(Y D)$.

Summing the last two equations gives

$$
\begin{aligned}
q^{\ell(Y)}\left\langle W\left|E Y C^{m}\right| V\right\rangle+\left\langle W\left|D Y C^{m}\right| V\right\rangle= & q^{\# E^{\prime} s \text { in } Y}\left\langle W\left|Y C^{m+1}\right| V\right\rangle \\
& + \text { terms of shorter length. }
\end{aligned}
$$

But now since the right-hand sides of equations (9) and (10) are known quantities, and the determinant of the coefficient matrix is $\alpha+\gamma q^{\ell(Y)}$ which is nonzero, we can solve for $\left\langle W\left|E Y C^{m}\right| V\right\rangle$ and $\left\langle W\left|D Y C^{m}\right| V\right\rangle$. This completes the proof.

Proposition 5.3. Suppose that $q \neq 1$. Let $D, E,\langle W|$, and $|V\rangle$ be as in Theorem 2.2, and set $C=D+E$. Let $c=J$ (recall that $J$ is given by 
Proposition 2.3, depending on three cases). Denote the length of $Y$ by $\ell(Y)$. For each word $Y$ in $D$ and $E$, define

$$
g(Y)=c^{\ell(Y)} \lim _{m \rightarrow \infty} \frac{\left\langle W\left|Y C^{m}\right| V\right\rangle}{\left\langle W\left|C^{m}\right| V\right\rangle} .
$$

Then $g(Y)$ satisfies the relations of Remark 4.4.

Proof. By Proposition 5.2, the definition of $g(Y)$ makes sense. Now note that the relations (a.) and (b.) of Remark 4.4 follow directly from relations (A.) and (B.) of Theorem 2.1. To check relation (c.), note that

$$
\begin{aligned}
g(Y(D+E)) & =c^{\ell(Y)+1} \lim _{m \rightarrow \infty} \frac{\left\langle W\left|Y(D+E) C^{m}\right| V\right\rangle}{\left\langle W\left|C^{m}\right| V\right\rangle} \\
& =c^{\ell(Y)+1} \lim _{m \rightarrow \infty} \frac{\left\langle W\left|Y C^{m+1}\right| V\right\rangle}{\left\langle W\left|C^{m}\right| V\right\rangle} \\
& =c^{\ell(Y)+1} \lim _{m \rightarrow \infty} \frac{\left\langle W\left|Y C^{m+1}\right| V\right\rangle}{\left\langle W\left|C^{m+1}\right| V\right\rangle} \cdot \frac{\left\langle W\left|C^{m+1}\right| V\right\rangle}{\left\langle W\left|C^{m}\right| V\right\rangle} \\
& =c^{\ell(Y)} \lim _{m \rightarrow \infty} \frac{\left\langle W\left|Y C^{m+1}\right| V\right\rangle}{\left\langle W\left|C^{m+1}\right| V\right\rangle} \\
& =g(Y) .
\end{aligned}
$$

Finally we turn to the proof of Theorem 5.1.

Proof. First note that if $q=1$ (hence $c=0$ ), it is easy to check that the measure defined by (8) is positive. This can be checked directly from the ansatz relations, which become very simple.

We now consider the case that $q<1$. By Proposition 5.3, we can define a function $g$ which satisfies the relations of Remark 4.4. Therefore the signed measure defined by $(8)$ is stationary for the process defined by $\mathcal{L}$. Moreover,

$$
\frac{\left\langle W\left|Y C^{m}\right| V\right\rangle}{\left\langle W\left|C^{\ell(Y)+m}\right| V\right\rangle}
$$

is non-negative, because it is a correlation function in the finite ASEP. Since $c$ is positive, and $g(Y)$ is a limit of non-negative values, it follows that $g(Y)$ is non-negative. Therefore the signed measure defined by (8) using the function $g$ from Proposition 5.3 is a positive measure.

We now need to check that $c$ satisfies one of the inequalities in Theorem 5.1 , and that indeed, any pair of $a$ and $c$ satisfying these inequalities can be obtained from Proposition 5.3 using a suitable choice of $\alpha, \beta, \gamma$, and $\delta$. 
Recall that $J$ was computed in Proposition 2.3, and that we have set $c=J$. Therefore in the first two cases, $c$ satisfies the inequalities of Theorem 5.1. We now consider the third case. If $a \geq 1$ and $b>a$ then it follows that

$$
c=J=\frac{(1-q) b}{(1+b)^{2}} \leq \frac{(1-q) a}{(1+a)^{2}} .
$$

While if $a \leq 1$ and $b>a$ then

$$
c=J=\frac{(1-q) b}{(1+b)^{2}} \leq \frac{1-q}{4} .
$$

In both cases, it follows that $c=J$ satisfies the inequalities of Theorem 5.1.

Moreoever, if we let $b$ tend to infinity (one may achieve this by sending $\beta$ to 0$)$, then $c=\frac{(1-q) b}{(1+b)^{2}}$ tends to 0 . Therefore it is possible to choose appropriate values $\alpha, \beta, \gamma, \delta$ and $q$ so as to realize any pair $(a, c)$ satisfying the conditions of Theorem 5.1. By the previous arguments, the corresponding measure that we get in this case is positive.

\section{Proof of Theorem 1.1}

In this section we prove Theorem 1.1.

Note that if we set $\beta=c$ and $\delta=-c$ in Theorem 2.1, then the relations (A.), (B.), and (C.) become:

(A'.) $D E-q E D=D+E$

(B'.) $\alpha\langle W| E-\gamma\langle W| D=\langle W|$

(C'.) $(D+E)|V\rangle=\frac{1}{c}|V\rangle$.

Note that these relations are nearly identical to the relations (a.), (b.) and (c.) from the Matrix Ansatz for the semi-infinite ASEP.

Proposition 6.1. Suppose that d,e, $\langle w|$ and $|v\rangle$ satisfy (a.), (b.), and (c.), and suppose that $D, E,\langle W|$, and $|V\rangle$ satisfy ( $A^{\prime}$.), ( $B^{\prime}$.), and ( $C^{\prime}$.). Let $y$ be an arbitrary word of length $\ell$ in $d$ and $e$, and let $Y$ be the corresponding word in $D$ and $E$. Then

$$
\frac{\langle w|y| v\rangle}{\left\langle w\left|(d+e)^{\ell}\right| v\right\rangle}=\frac{\langle W|Y| V\rangle}{\left\langle W\left|(D+E)^{\ell}\right| V\right\rangle} .
$$

Proof. Let $\widetilde{D}=c D$ and $\widetilde{E}=c E$. Since $D, E,\langle W|$, and $V\rangle$ satisfy (A'.), (B'.), and (C'.), it is easy to verify that $\widetilde{D}, \widetilde{E},\langle W|$, and $V\rangle$ satisfy (a.), (b.), and (c.). We also have that $d, e,\langle w|$, and $|v\rangle$ satisfy (a.), (b.), and (c.). 
Therefore both of them yield the same measure, as defined in Theorem 4.1. Letting $\widetilde{Y}$ denote the word in $\widetilde{D}$ and $\widetilde{E}$ corresponding to $Y$, we have that

$$
\frac{\langle w|y| v\rangle}{\left\langle w\left|(d+e)^{\ell}\right| v\right\rangle}=\frac{\langle W|\widetilde{Y}| V\rangle}{\left\langle W\left|(\widetilde{D}+\widetilde{E})^{\ell}\right| V\right\rangle}=\frac{\langle W|Y| V\rangle}{\left\langle W\left|(D+E)^{\ell}\right| V\right\rangle} .
$$

Theorem 1.1 now follows from Proposition 6.1, and Theorems 4.1 and 2.1 .

\section{Conclusion}

In this paper we have given a combinatorial interpretation for the stationary measures of the semi-infinite ASEP. More specifically, one may compute the finite correlation functions of the stationary measures using sums over staircase tableaux, with the parameters $\alpha, \beta=c, \gamma, \delta=-c$, and $q$.

In particular, we have demonstrated that a rather nonsensical specialization of the stationary distribution of the finite ASEP - the specialization $\delta=-\beta$ - can be given a meaningful interpretation in terms of the ASEP on a semi-infinite lattice.

One might ask more generally when this phenomenon can occur. For concreteness, in the discussion below, we will consider finite Markov chains.

- Consider a Markov chain $M$ whose transition matrix is written in terms of one or more parameters (e.g. hopping rates). Typically we don't consider $M$ to "make sense" unless these parameters are nonnegative.

- Recall that the stationary distribution $\mu$ of a Markov chain is the unique left eigenvector of the transition matrix associated with eigenvalue 1.

- One may choose a specialization of the parameters and consider the corresponding specialization of $\mu$.

- If one makes one or more parameters negative (or even complex), when can one still give a probabilistic or physical meaning to the corresponding "stationary distribution," that is, the corresponding specialization of $\mu$ ?

\section{References}

[1] S. Corteel, R. Stanley, D. Stanton, L. Williams, Formulae for AskeyWilson moments and enumeration of staircase tableaux, Trans. Amer. Math. Soc. 364 (2012), 6009-6037. MR2946941 
[2] S. Corteel, L. Williams, Staircase tableaux, the asymmetric exclusion process, and Askey-Wilson polynomials, Proc. Natl. Acad. Sci. (2010), doi:10.1073/pnas.0909915107. MR2630104

[3] S. Corteel, L. Williams, Tableaux combinatorics for the asymmetric exclusion process and Askey-Wilson polynomials, Duke Math. J. 159 (2011) 385-415. MR2831874

[4] B. Derrida, M. Evans, V. Hakim, V. Pasquier, Exact solution of a 1D asymmetrix exclusion model using a matrix formulation, J. Phys. A: Math. Gen. 26 (1993), 1493-1517. MR1219679

[5] B. Derrida, J. Lebowitz, E. Speer, J. Stat. Phys. 89 (1997), 135-167. MR1492490

[6] S. Grosskinsky, Ph.D. Dissertation, Technical University of Munich, 2004 .

[7] T. Liggett, Ergodic theorems for the asymmetric simple exclusion process, Trans. Amer. Math. Soc. 213 (1975), 237-261. MR0410986

[8] J. MacDonald, J. Gibbs, A. Pipkin, Biopolymers 6 (1968), no. 1.

[9] F. Spitzer, Interaction of Markov processes, Adv. Math. 5 (1970), 246290. MR0268959

[10] M. Uchiyama, T. Sasamoto, M. Wadati, Asymmetric simple exclusion process with open boundaries and Askey-Wilson polynomials, J. Phys. A 37 (2004), no. 18. MR2065218

TOMOHIRo SASAMOTO

Department of Mathematics

ChIBA UNIVERSITY

YAYOI-CHO 1-33, INAGE

CHIBA 263-8522

JAPAN

E-mail address: sasamoto@math.s.chiba-u.ac.jp

LAUREN WILLIAMS

Department of Mathematics

EVANS HaLl 913

UNIVERSITY OF CALIFORNIA

BERKELEY

USA

E-mail address: williams@math. berkeley.edu

RECEIVED 19 MAY 2014 